M. Yamazato

Nagoya Math. J.

Vol. 127 (1992), 175-200

\title{
ON SUBCLASSES OF INFINITELY DIVISIBLE \\ DISTRIBUTIONS ON $R$ RELATED TO HITTING \\ TIME DISTRIBUTIONS OF 1-DIMENSIONAL GENERALIZED DIFFUSION PROCESSES
}

\author{
MAKOTO YAMAZATO
}

\section{Introduction}

A distribution $\mu$ on $\mathbf{R}_{+}=[0, \infty)$ is said to be a $C M E_{+}^{f}$ distribution if there are an increasing (in the strict sense) sequence of positive real numbers $\left\{a_{k}\right\}_{k=1}^{\ell}$ and $0=b_{0}<b_{1}<\cdots<b_{m}<b_{m+1}=\infty(0 \leqq m<\ell<\infty)$ such that, for each $j=0, \ldots, m$, there is at least one $a_{k}$ satisfying $b_{j}<a_{k}<b_{j+1}$ and the Laplace transform $\mathscr{L} \mu(s)=\int_{\mathbf{R}_{+}} e^{-s x} \mu(d x)$ of $\mu$ is represented as

$$
\begin{aligned}
\mathscr{L} \mu(s) & =\prod_{i=1}^{\ell} a_{i}\left(s+a_{i}\right)^{-1} & & \text { if } m=0, \\
& =\prod_{i=1}^{\ell} a_{i}\left(s+a_{i}\right)^{-1} / \prod_{j=1}^{\ell} b_{j}\left(s+b_{j}\right)^{-1} & & \text { if } m \geqq 1 .
\end{aligned}
$$

The author [8] shows that the upward first passage time distributions of birth and death processes belong to the class $C M E_{+}^{f}$. He [9] also shows that the class of distributions of hitting times of single points of generalized diffusion processes is a proper subclass of the closure $C M E_{+}$, in the weak convergence sense, of $C M E_{+}^{f}$. Let $C M E_{-}^{f}$ be the class of distributions on $\mathbf{R}_{-}=(-\infty, 0]$ whose mirror images belong to $C M E_{+}^{f}$. That is, $\mu \in C M E_{-}^{f}$ if and only if $\bar{\mu}(d u)=\mu(-d u)$ belongs to $C M E_{+}^{f}$. Let $C M E^{f}$ be the class of $\mu=\mu_{1} * \mu_{2}$ with $\mu_{1} \in C M E_{+}^{f}$ and $\mu_{2} \in C M E_{-}^{f}$. Sato [4] shows that the distributions of sojourn times of birth and death processes with weight not necessarily positive belong to $C M E^{f}$.

We denote the class of infinitely divisible distributions on $\mathbf{R}$ (or $\mathbf{R}_{ \pm}$) by $\mathscr{I}(\mathbf{R})$ (or $\mathscr{I}\left(\mathbf{R}_{ \pm}\right)$). The classes $C M E_{+}^{f}$ and $C M E_{+}$are contained in $\mathscr{I}\left(\mathbf{R}_{+}\right)$. The class $C M E^{f}$ is contained in $\mathscr{I}(\mathbf{R})$. Some interesting classes of infinitely divisible distributions on $\mathbf{R}_{+}$(for example, $B O, C E_{+}, M E_{+}, C M E_{+}, \ldots$ ) are introduced in [1] and [8] and representations of their Laplace transforms, compactness

$\overline{\text { Received April }}$ 12, 1991. 
conditions and convergence conditions are investigated. Sato's result [4] suggests that it is natural to extend those classes to classes on $\mathbf{R}$. We denote by $B_{+}$the class $B O$ in this paper.

The main purpose of this paper is to define classes $B, C E, M E, C M E$ on $\mathbf{R}$, obtain representations of their characteristic functions or Laplace transforms, and express convergence conditions by their characteristics. This will be done in Sections $2 \sim 5$. Thorin [6] extended the notion of generalized $\Gamma$-convolutions on the half real line, which is a natural subclass of $B_{+}$and class $L$ containing the class of stable distributions and the class $C E_{+}$, to those on the whole real line and gets a convergence condition (parallel to our Theorem 2.1). In Section 6, we define and study a subclass $M E_{+}^{d}$ of $M E_{+}$and a subclass $C M E_{+}^{d}$ of $C M E_{+}$. It is shown in [9] that hitting time distributions of one dimensional generalized diffusion processes with non-natural boundaries belong to the class $C M E_{+}^{d}$.

In the naming of the classes, $C, M$, and $E$ suggest convolution, mixture, and exponential distributions, respectively. The superscripts $f$ and $d$ suggest finite and discrete, respectively.

Necessary and sufficient condition for strong unimodality for a subclass of $C M E_{+}$is given in [7]. An extension of the result to $C M E$ will be given in [10].

Acknowledgement. The author would like to express his hearty thanks to the referee for his valuable comments. He also thanks Ken-iti Sato for his useful advices.

\section{Class $B$}

For a topological space $A$, we denote by $\mathscr{P}(A)$ the totality of Borel probability measures on $A$. For $\mu_{1}, \mu_{2} \in \mathscr{P}(\mathbf{R})$, we denote by $\mu_{1} * \mu_{2}$ the convolution of $\mu_{1}$ and $\mu_{2}$. For $A, B \subset \mathscr{P}(\mathbf{R})$, we denote by $A * B$ the totality of $\mu=\mu_{1} * \mu_{2}$ with $\mu_{1} \in$ $A$ and $\mu_{2} \in B$. The characteristic function of $\mu \in \mathscr{P}(\mathbf{R})$ is denoted by $\mathscr{F} \mu(s)$. We define the bilateral Laplace transform $\mathscr{L} \mu(s)=\int_{\mathbf{R}} e^{-s x}(d x)$ if the integral is finite. A representation of the characteristic functions of infinitely divisible distributions is well known. Namely, a distribution $\mu \in \mathscr{P}(\mathbf{R})$ is infinitely divisible if and only if there are $\gamma \in \mathbf{R}, \sigma>0$ and a measure $\nu$ on $\mathbf{R}_{0}=\mathbf{R} \backslash\{0\}$ satisfying

$$
\int_{\mathbf{R}_{0}}\left(x^{2} \wedge 1\right) \nu(d x)<\infty
$$


such that

$$
\begin{aligned}
& \mathscr{F} \mu(z) \\
= & \exp \left\{i \gamma z-\sigma^{2} z^{2} / 2+\int_{\mathbf{R}_{0}}\left(e^{i z x}-1-\frac{i z x}{1+x^{2}}\right) \nu(d x)\right\} .
\end{aligned}
$$

Here, $a \wedge b=\min \{a, b\}$. This representation is unique. We call (2.2) the canonical representation $\left[\gamma, \sigma^{2}, \nu\right]$ of $\mu \in \mathscr{I}(\mathbf{R})$. The measure $\nu$ is called Lévy measure of $\mu$. The following theorem is well known.

THEOREM A. Let $\mu_{n} \in \mathscr{I}(\mathbf{R})$ with canonical representation $\left[\gamma_{n}, \sigma_{n}, \nu_{n}\right]$ and let $\mu \in \mathscr{P}(\mathbf{R})$. Then the following (i) and (ii) are equivalent:

(i) $\mu_{n}$ converges weakly to $\mu$ as $n \rightarrow \infty$.

(ii) $\mu$ is infinitely divisible. Let $[\gamma, \sigma, \nu]$ be its canonical representation.

(a) For every bounded continuous function $f$ which vanishes near the origin,

$$
\int f(u) \nu_{n}(d u) \rightarrow \int f(u) \nu(d u) \text { as } n \rightarrow \infty
$$

(b) For $\varepsilon>0$ set

$$
A_{n, \varepsilon}=\sigma_{n}^{2}+\int_{|y|<\varepsilon} y^{2} \nu_{n}(d u)
$$

Then

$$
\lim _{\varepsilon \downarrow 0} \limsup _{n \rightarrow \infty} A_{n, \varepsilon}=\lim _{\varepsilon \downarrow 0} \liminf _{n \rightarrow \infty} A_{n, \varepsilon}=\sigma^{2} .
$$

$$
\lim _{n \rightarrow \infty} \gamma_{n}=\gamma \text {. }
$$

We say that a distribution $\mu$ on $\mathbf{R}$ is a $B$ distribution if $\mu \in \mathscr{I}(\mathbf{R})$ and its Lévy measure $\nu$ is absolutely continuous with density $\ell$ represented as

$$
\begin{aligned}
\ell(y) & =\int_{(0, \infty)} e^{-y u} Q(d u) \text { for } y>0, \\
& =\int_{(-\infty, 0)} e^{-y u} Q(d u) \text { for } y<0,
\end{aligned}
$$

where, $Q$ is a measure on $\mathbf{R}_{0}$ satisfying

$$
\int_{\mathbf{R}_{0}}|u|^{-1} \wedge|u|^{-3} Q(d u)<\infty
$$

We denote by $B_{+}$the class of $B$ distributions on $R_{+}$. The class $B_{+}$here was denoted by $B O$ in [8] and called g.c.m.e.d. (generalized convolutions of mixtures 
of exponential distributions) in [1]. The above integrability condition (2.3) for $Q$ is equivalent to the condition (2.1) for $\nu$. We call $Q$ the $Q$-measure of $\mu \in B$. A $B$ distribution $\mu$ is uniquely represented by the triplet $\left(\gamma, o^{2}, Q\right)$. We describe a necessary and sufficient condition for weak convergence in $B$ in terms of this triplet.

THEOREM 2.1. Let $\mu_{n} \in B$ and let $\left(\gamma_{n}, \sigma_{n}^{2}, Q_{n}\right)$ be its triplet. In order that $\mu_{n}$ converges to $\mu \in \mathscr{P}(\mathbf{R})$ as $n \rightarrow \infty$, it is necessary and sufficient that $\mu \in B$ with triplet $\left(\gamma, \sigma^{2}, Q\right)$ and the following conditions are satisfied.

(i) For any function $f$ with compact support in $\mathbf{R}$ such that $|u| f(u)$ is continuous,

$$
\int f(u) Q_{n}(d u) \rightarrow \int f(u) Q(d u) \text { as } n \rightarrow \infty .
$$

(ii) Let $A_{n, M}=\sigma_{n}^{2}+2 \int_{|u|>M}|u|^{-3} Q_{n}(d u)$. Then

$$
\lim _{M \rightarrow \infty} \limsup _{n \rightarrow \infty} A_{n, M}=\lim _{M \rightarrow \infty} \liminf _{n \rightarrow \infty} A_{n, M}=\sigma^{2} .
$$

(iii)

$$
\lim _{n \rightarrow \infty} \gamma_{n}=\gamma
$$

Proof. We prove the theorem checking the conditions of Theorem A.

Sufficiency. Assume that $\mu \in B$ and (i) $\sim$ (iii) hold. Let $\nu_{n}$ and $\nu$ be the Lévy measures of $\mu_{n}$ and $\mu$, respectively. By (i) and (ii), we have

$$
\int f(u)\left(|u|^{-1} \wedge|u|^{-3}\right) Q_{n}(d u) \rightarrow \int f(u)\left(|u|^{-1} \wedge|u|^{-3}\right) Q(d u)
$$

as $n \rightarrow \infty$ for every continuous function $f$ on $\mathbf{R}$ vanishing at infinity. Hence for $0<a<b$

$$
\begin{gathered}
\int_{a}^{b} \nu_{n}(d y)=\int_{0}^{\infty} u^{-1}\left(e^{-a u}-e^{-b u}\right) Q_{n}(d u) \\
\rightarrow \int_{0}^{\infty} u^{-1}\left(e^{-a u}-e^{-b u}\right) Q(d u) \\
=\int_{a}^{b} \nu(d y) \text { as } n \rightarrow \infty .
\end{gathered}
$$

In the same manner,

$$
\int_{1}^{\infty} \nu_{n}(d y)=\int_{0}^{\infty} u^{-1} e^{-u} Q_{n}(d u) \rightarrow \int_{1}^{\infty} \nu(d y),
$$




$$
\begin{gathered}
\int_{-b}^{-a} \nu_{n}(d y) \rightarrow \int_{-b}^{-a} \nu(d y), \\
\int_{-\infty}^{-1} \nu_{n}(d y) \rightarrow \int_{-\infty}^{-1} \nu(d y) \text { as } n \rightarrow \infty .
\end{gathered}
$$

Hence we get the condition (a) in Theorem A. Note that

$$
\begin{aligned}
\int_{|y|<\varepsilon} y^{2} \nu_{n}(d y) & =\int_{\mathbf{R}_{0}}\left(\int_{0}^{\varepsilon} y^{2} e^{-|u| y} d y\right) Q_{n}(d u) \\
& =\int_{\mathbf{R}_{0}}\left(\int_{0}^{|u| \varepsilon} y^{2} e^{-y} d y\right)|u|^{-3} Q_{n}(d u) \\
& =\sum_{i=1}^{4} F_{n}^{i}(\varepsilon)
\end{aligned}
$$

where, for $\varepsilon>0$,

$$
\begin{aligned}
& F_{n}^{1}(\varepsilon)=\int_{|u| \geq \varepsilon^{-2}} 2|u|^{-3} Q_{n}(d u), \\
& F_{n}^{2}(\varepsilon)=-\int_{|u| \geqq \varepsilon^{-2}}\left(\int_{|u| \varepsilon}^{\infty} y^{2} e^{-y} d y\right)|u|^{-3} Q_{n}(d u), \\
& F_{n}^{3}(\varepsilon)=\int_{|u| \leq \varepsilon^{-2}}\left(\int_{0}^{\varepsilon} y^{2} e^{-|u| y} d y\right) Q_{n}(d u) .
\end{aligned}
$$

By (ii), we have that $\left\{F_{n}^{1}(\varepsilon)\right\}$ is bounded in $n$ and

$$
\left|F_{n}^{2}(\varepsilon)\right| \leqq \frac{1}{2} F_{n}^{1}(\varepsilon) \int_{\varepsilon^{-1}}^{\infty} y^{2} e^{-y} d y \rightarrow 0 \text { as } n \rightarrow 0 \text { and } \varepsilon \rightarrow 0 .
$$

In the following, we may assume that $\varepsilon^{-2}$ is a continuity point of $Q$. By (i), we have, for fixed $\varepsilon>0$,

$$
\lim _{n \rightarrow \infty} F_{n}^{3}(\varepsilon)=\int_{|u| \leqslant \varepsilon^{-2}}\left(\int_{0}^{\varepsilon} y^{2} e^{-|u| y} d y\right) Q(d u) .
$$

By (2.3) and by bounded convergence theorem,

$$
\begin{aligned}
& \int_{|u| \leqslant \varepsilon^{-2}}\left(\int_{0}^{\varepsilon} y^{2} e^{-|u| y} d y\right) Q(d u) \\
= & \int_{|u| \leqslant 1}\left(\int_{0}^{\varepsilon} y^{2} e^{-|u| y} d y\right) Q(d u)+\int_{1 \leq|u| \leq \varepsilon^{-2}}\left(\int_{0}^{|u| \varepsilon} y^{2} e^{-y} d y\right)|u|^{-3} Q(d u) \rightarrow 0 \text { as } \varepsilon \rightarrow 0 .
\end{aligned}
$$

Thus, we have

$\lim _{\varepsilon \downarrow 0} \lim _{n \rightarrow \infty} F_{n}^{3}(\varepsilon)=0$ 
Hence, we have

$$
\begin{aligned}
& \lim _{\varepsilon \downarrow 0} \limsup _{n \rightarrow \infty}\left[\sigma_{n}^{2}+\int_{|y|<\varepsilon} y^{2} \nu_{n}(d y)\right] \\
= & \lim _{M \uparrow \infty} \limsup _{n \rightarrow \infty}\left[\sigma_{n}^{2}+\int_{|u|>M} 2|u|^{-3} Q(d u)\right]
\end{aligned}
$$

and

$$
\begin{aligned}
& \lim _{\varepsilon \downarrow 0} \liminf _{n \rightarrow \infty}\left[\sigma_{n}^{2}+\int_{|y|<\varepsilon} y^{2} \nu_{n}(d y)\right] \\
= & \lim _{M \uparrow \infty} \liminf _{n \rightarrow \infty}\left[\sigma_{n}^{2}+\int_{|u|>M} 2|u|^{-3} Q(d u)\right] .
\end{aligned}
$$

Thus the condition (b) of Theorem A holds. The condition (c) is trivial.

Necessity. Let $\mu_{n} \rightarrow \mu$. Then $\mu \in \mathscr{I}(\mathbf{R})$ by Theorem A. By Theorem A(a), we have, for any continuity point $a>0$ of $\nu$.

$$
\int_{a}^{\infty} \nu_{n}(d u) \rightarrow \int_{a}^{\infty} \nu(d u) \text { as } n \rightarrow \infty
$$

Hence we have, for a.e. $a>0$,

$$
\int_{0}^{\infty} u^{-1} e^{-a u} Q_{n}(d u) \rightarrow \int_{a}^{\infty} \nu(d u) \text { as } n \rightarrow \infty
$$

Similarly we have, for a.e. $a<0$,

$$
\int_{-\infty}^{0}|u|^{-1} e^{-a u} Q_{n}(d u) \rightarrow \int_{-\infty}^{a} \nu(d u) \text { as } n \rightarrow \infty .
$$

By (2.4) we have,

$$
\int_{|y|<\varepsilon} y^{2} \nu_{n}(d y) \geqq F_{n}^{1}(\varepsilon)\left(1-2^{-1} \int_{\varepsilon^{-1}}^{\infty} y^{2} e^{-y} d y\right) .
$$

Thus $\left\{F_{n}^{1}(\varepsilon)\right\}$ is bounded in $n$. Then we see, by (2.7) and (2.8), that there is a finite measure $\widetilde{Q}$ on $\mathbf{R}$ such that for $a>0$

$$
\begin{gathered}
\int_{0}^{\infty} u^{-1} e^{-a u} Q_{n}(d u) \rightarrow \int_{0}^{1} e^{-a u} \widetilde{Q}(d u)+\int_{1}^{\infty} u^{2} e^{-a u} \widetilde{Q}(d u), \\
\int_{-\infty}^{0}|u|^{-1} e^{-a|u|} Q_{n}(d u) \rightarrow \int_{-1}^{0} e^{-a|u|} \widetilde{Q}(d u)+\int_{-\infty}^{-1} u^{2} e^{-a|u|} \widetilde{Q}(d u)
\end{gathered}
$$

as $n \rightarrow \infty$. Note that $\widetilde{Q}$ does not have a point mass at $\{0\}$ since $\lim _{a \rightarrow \infty} \int_{|y|>a} \nu(d y)$ $=0$. Set $Q(d u)=\left(|u| \vee|u|^{3}\right) \widetilde{Q}(d u)$. Then, $Q$ is a measure on $\mathbf{R}_{0}$ satisfying 
(2.3). We have

$$
\int_{I}|u|^{-1} Q_{n}(d u) \rightarrow \int_{I}|u|^{-1} Q(d u) \text { as } n \rightarrow \infty
$$

for every finite interval $I$ in $\mathbf{R}$ both end points of which are continuity points of $Q$. Thus, (i) holds. We have, by (i),

$$
\lim _{\varepsilon \downarrow 0} \lim _{n \rightarrow \infty} F_{n}^{3}(\varepsilon)=0 .
$$

Since $\left\{F_{n}^{1}\{\varepsilon)\right\}$ is bounded in $n$,

$$
\lim _{\varepsilon \downarrow 0} \limsup _{n \rightarrow \infty}\left|F_{n}^{2}(\varepsilon)\right|=0 .
$$

We have (2.5) and (2.6). Hence (ii) holds. The proof is complete.

COROllary. The class $B$ is closed under convolution and weak convergence.

THEOREM 2.2. The class $B$ coincides with the closure of $B_{+} * B_{-}$.

Proof. Since the class $B$ is closed, it is enough to show that the normal distributions and $B$ distributions without Gaussian components are approximated by $B_{+} * B_{-}$distributions. For $\sigma^{2}>0$, set $\alpha_{n}=\left(2 n / \sigma^{2}\right)^{1 / 2}$ and let

$$
\begin{aligned}
q_{n}(x) & =0 \text { for }|x|<\alpha_{n}, \\
& =n \text { for } a_{n} \leqq|x| .
\end{aligned}
$$

Then $\mu_{n}=\left(0,0, q_{n}(x) d x\right) \in B_{+} * B_{-}$. We have, for $M<\alpha_{n}$

$$
2 \int_{|u|>M}|u|^{-3} q_{n}(u) d u=\sigma^{2}
$$

and for every finite interval $I$ in $\mathbf{R}$,

$$
\int_{I}|u|^{-1} q_{n}(u) d u \rightarrow 0
$$

as $n \rightarrow \infty$. Hence $\mu_{n} \rightarrow\left(0, \sigma^{2}, 0\right)$ as $n \rightarrow \infty$ by Theorem 2.1. Now, let $(0,0, Q)$ $\in B$. Define $Q_{n}$ by $Q_{n}=\left.Q\right|_{[-n, n]}$. Then $\left(0,0, Q_{n}\right) \in B_{+} * B_{-}$.

Since

$$
\int_{|u|>M}|u|^{-3} Q_{n}(d u) \rightarrow \int_{|u|>M}|u|^{-3} Q(d u)
$$


as $n \rightarrow \infty$ and

$$
\int_{|u|>M}|u|^{-3} Q(d u) \rightarrow 0
$$

as $M \rightarrow \infty,\left(0,0, Q_{n}\right) \rightarrow(0,0, Q)$. The proof is complete.

\section{Class $M E$}

We say that a probability distribution $\mu$ on $\mathbf{R}_{+}$is an $M E_{+}$distribution if there is a probability measure $G$ on $(0, \infty]$ such that

$$
\begin{aligned}
\mu[0, x] & =G(\{\infty\}) & \text { if } & x=0, \\
& =\int_{(0, \infty)}\left(1-e^{-x u}\right) G(d u) & \text { if } & x>0,
\end{aligned}
$$

where the value of the integrand $1-e^{-x u}$ at infinity for $x>0$ is defined by its limit 1 as $u \rightarrow \infty$. We call $G$ the mixing distribution of $\mu$. We denote by $M E_{+}$the class of $M E_{+}$distributions. It is easy to see that the Laplace transform of $\mu \in M E_{+}$is represented by its mixing distribution $G$ as:

$$
\begin{aligned}
\mathscr{L} \mu(s) & =G(\{\infty\})+\int_{(0, \infty)} e^{-s x} d x \int_{(0, \infty)} u e^{-x u} G(d u) \\
& =\int_{(0, \infty)} \frac{u}{s+u} G(d u) .
\end{aligned}
$$

Define $M E_{-}$by the mirror image of $M E_{+}$. That is, $M E_{-}$if and only if $\mu \in$ $\mathscr{P}\left(\mathbf{R}_{-}\right)$and

$$
\begin{array}{rlrl}
\mu[x, 0] & =G(\{-\infty\}) & & \text { if } \quad x=0 \\
& =\int_{[-\infty, 0)}\left(1-e^{-x u}\right) G(d u) & \text { if } \quad x<0
\end{array}
$$

with $G \in \mathscr{P}([-\infty, 0))$. Let $M E=M E_{+} * M E_{-}$. A representation of the Laplace transform of $\mu \in M E_{+}$is obtained by Steutel [5]. We state here his representation.

THEOREM B. A probability measure $\mu$ on $\mathbf{R}_{+}$is an $M E_{+}$distribution if and only if there is a nonnegative and absolutely continuous measure $Q$ on $R_{+}$with density bounded by 1 a.e. satisfying $\int_{0}^{1} u^{-1} Q(d u)<\infty$ such that, for $z \in \mathbf{R}$,

$$
\mathscr{F} \mu(z)=\exp \left[\int_{\mathbf{R}_{+}}\left(e^{i z x}-1\right)\left(\int_{\mathbf{R}_{+}} e^{-x u} Q(d u)\right) d x\right]
$$


By this theorem, we easily get the representation of the characteristic function of $\mu \in M E$ :

$$
\mathscr{F} \mu(z)=\exp \left[\int_{\mathbf{R}_{0}}\left(e^{t z x}-1\right) \ell(x) d x\right]
$$

where

$$
\begin{aligned}
& \ell(x)=\int_{\mathbf{R}_{+}} e^{-x u} Q(d u) \text { for } x>0 \text {, } \\
& =\int_{\mathbf{R}_{-}} e^{-x u} Q(d u) \text { for } x<0
\end{aligned}
$$

and $Q$ is an absolutely continuous measure on $\mathbf{R}$ with density bounded by 1 a.e. satisfying

$$
\int_{|u|<1}|u|^{-1} Q(d u)<\infty
$$

Hence $M E \subset B$ and the above $Q$ is the $Q$-measure of $\mu$.

Remark 3.1. Let $\mu \in M E_{+}$and let $G$ be its mixing distribution. Let $\ell$ be the density of the Lévy measure of $\mu$ and let $Q$ be the $Q$-measure of $\mu$. Then

$$
\begin{aligned}
G(\{\infty\}) & =\exp \left\{-\int_{0}^{\infty} \ell(x) d x\right\} \\
& =\exp \left\{-\int_{0}^{\infty} \frac{1}{u} Q(d u)\right\} .
\end{aligned}
$$

Proof. It is easy to see that

$$
G(\{\infty\})=\lim _{s \rightarrow \infty} \mathscr{L} \mu(s)=\exp \left\{-\int_{0}^{\infty} \ell(x) d x\right\} .
$$

Since

$$
\begin{aligned}
\int_{0}^{\infty} \ell(x) d x & =\int_{0}^{\infty}\left(\int_{0}^{\infty} e^{-u x} d x\right) Q(d u) \\
& =\int_{0}^{\infty} \frac{1}{u} Q(d x\},
\end{aligned}
$$

we get the conclusion.

THEOREM 3.1. Let $\mu_{n} \in M E_{+}$and $\mu \in \mathscr{P}\left(\mathbf{R}_{+}\right)$. Let $G_{n}$ be the mixing distribution of $\mu_{n}$. Then $\mu_{n}$ converges weakly to $\mu$ if and only if $\mu \in M E_{+}$and $G_{n}$ converges weakly to $G$, the mixing distribution of $\mu$, as a sequence of distributions on $(0, \infty]$ as $n \rightarrow \infty$. 
Proof. Let $F_{n}$ and $F$ be the distribution functions of $\mu_{n}$ and $\mu$, respectiveiy. Assume that $\mu \in M E_{+}$and $G_{n} \rightarrow G$ weakly on $(0, \infty]$ as $n \rightarrow \infty$. Then, obviously we have, for $x>0$,

$$
\begin{aligned}
F_{n}(x) & =\int_{(0, \infty)}\left(1-e^{-x u}\right) G_{n}(d u) \\
\rightarrow F(x) & =\int_{(0, \infty)}\left(1-e^{-x u}\right) G(d u) \quad \text { as } n \rightarrow \infty .
\end{aligned}
$$

This shows that $\mu_{n} \rightarrow \mu$. Conversely, we assume that $\mu_{n} \rightarrow \mu$ weakly as $n \rightarrow \infty$. Then we have $F_{n}(x) \rightarrow F(x)$ as $n \rightarrow \infty$ for all continuity point $x>0$. For $\varepsilon>0$, we can choose $x>0$ sufficiently large so that $1-F_{n}(x)<\varepsilon$ for all $n$. Hence,

$$
e^{-x \delta} G_{n}(0, \delta) \leqq \int_{(0, \delta)} e^{-x u} G_{n}(d u)<\varepsilon
$$

i.e.

$$
G_{n}(0, \delta)<\varepsilon e^{x \delta}
$$

This means that $\left\{G_{n}\right\}$ is a conditionally compact sequence as measures on $(0, \infty]$. Choosing subsequence $\left\{n^{\prime}\right\}$ of $\{n\}$ so that $G_{n}$, converges to a distribution $G$ on $(0, \infty]$, we have

$$
\begin{aligned}
F_{n^{\prime}}(x) & =\int_{(0, \infty]}\left(1-e^{-x u}\right) G_{n^{\prime}}(d u) \\
& \rightarrow \int_{(0, \infty]}\left(1-e^{-x u}\right) G(d u) \quad \text { as } n^{\prime} \rightarrow \infty
\end{aligned}
$$

for $x>0$. Hence

$$
F(x)=\int_{(0, \infty]}\left(1-e^{-x u}\right) G(d u)
$$

for continuity point $x$ of $F$. Since the right hand side is continuous for $x>0$ and since $F$ is right continuous, the equality holds for all $x>0$. Letting $x \rightarrow 0$, we get $F(0)=G(\{\infty\})$. Hence

$$
F(x)=1-\int_{(0, \infty)} e^{-x u} G(d u) .
$$

By the uniqueness for Laplace transforms, $G_{n}$ converges weakly to $G$ on $(0, \infty]$ as $n \rightarrow \infty$. The proof is complete.

Theorem 3.2. Let $\mu_{+} \in M E_{+}, \mu_{-} \in M E_{-}$and let $\mu=\mu_{+} * \mu_{-} \in M E$. Then $\mu$ is absolutely continuous on $\mathbf{R}_{0}$ and has a point mass $\mu_{+}(\{0\}) \mu_{-}(\{0\})$ at the origin. 
Let $h$ be the density of $\mu$ on $\mathbf{R}_{0}$. Let $G_{+}$and $G_{-}$be mixing distributions of $\mu_{+}$and $\mu_{-}$, respectively. Denote $\psi_{+}(s)=\mathscr{L} \mu_{+}(s)$ and $\phi_{-}(s)=\mathscr{L}_{\mu_{-}}(s)$. Then the following hold:

$$
\begin{aligned}
h(x) & =\left(h_{+} * h_{-}\right)(x)+\mu_{-}(\{0\}) h_{+}(x) \\
& =\int_{(0, \infty)} \phi_{-}(-u) u e^{-u x} G_{+}(d u) \text { for } x>0,
\end{aligned}
$$

and

$$
\begin{aligned}
h(x) & =\left(h_{+} * h_{-}\right)(x)+\mu_{+}(\{0\}) h_{-}(x) \\
& =\int_{(-\infty, 0)} \phi_{+}(-v)|v| e^{-v x} G_{-}(d v) \text { for } x<0,
\end{aligned}
$$

where $h_{+}$and $h_{-}$are densities of $\mu_{+}$and $\mu_{-}$on $(0, \infty)$ and $(-\infty, 0)$, respectively.

(ii) Denote $d_{-}=\sup \left\{v<0 ; G_{-}([v, 0))>0\right\}$ and $d_{+}=\inf \left\{v>0 ; G_{+}((0, v])\right.$ $>0\}$. If $d_{-}<d_{+}$, then the Laplace transform $\mathscr{L} \mu(s)$ of $\mu$ exists for $-d_{+}<s<$ $-d_{-}$and is represented as

$$
\begin{aligned}
& \mathscr{L} \mu(s)=\int_{(-\infty, 0)} \phi_{+}(-v) \frac{v}{s+v} G_{-}(d v)+ \\
& \quad+\int_{(0, \infty)} \psi_{-}(-u) \frac{u}{s+u} G_{+}(d u)+G_{+}(\{\infty]) G_{-}(\{-\infty\}) .
\end{aligned}
$$

Proof. (i) Let $F, F_{+}$and $F_{-}$be the distribution functions of $\mu, \mu_{+}$and $\mu_{-}$, respectively. Let $x>0$. Then,

$$
\begin{aligned}
& F(x) \\
= & \int_{(-\infty, 0)} F_{+}(x-y) F_{-}(d y)+F_{+}(x) \mu_{-}(\{0\}) \\
= & \int_{(-\infty, 0)} h_{-}(y) d y \int_{0}^{x-y} h_{+}(z) d z+\mu_{-}(\{0\})\left(\int_{0}^{x} h_{+}(z) d z+\mu_{+}(\{0\})\right) .
\end{aligned}
$$

By this we get

$$
h(x)=\int_{(-\infty, 0)} h_{+}(x-y) h_{-}(y) d y+\mu_{-}(\{0\}) h_{+}(x) \text { for } \quad x>0 .
$$

By the definition of the classes $M E_{+}$and $M E_{-}$we have

$$
\int_{(-\infty, 0)} h_{+}(x-y) h_{-}(y) d y
$$




$$
\begin{aligned}
& =\int_{(-\infty, 0)}\left(\int_{(0, \infty)} u e^{-u(x-y)} G_{+}(d u)\right)\left(\int_{(-\infty, 0)}|v| e^{-v y} G_{-}(d v)\right) d y \\
& =\int_{(0, \infty)} G_{+}(d u) \int_{(-\infty, 0)} \frac{v u}{v-u} e^{-u x} G_{-}(d v) \\
& =\int_{(0, \infty)}\left(\int_{(-\infty, 0)} \frac{v}{v-u} G_{-}(d v)\right) u e^{-u x} G_{+}(d u) .
\end{aligned}
$$

Thus,

$$
h(x)=\int_{(0, \infty)} \phi_{-}(-u) u e^{-u x} G_{+}(d u)<\infty .
$$

In the same way we get the representation for $x<0$.

(ii) If $-d_{+}<s<-d_{-}$, the right hand side of (3.3) is well defined. Denote by $A(s)$ the right hand side of (3.3). Set

$$
\widetilde{\phi}_{-}(s)=\int_{(-\infty, 0)} \frac{v}{s+v} G_{-}(d v)
$$

and

$$
\widetilde{\phi}_{+}(s)=\int_{(0, \infty)} \frac{u}{s+u} G_{+}(d u) .
$$

Note that, by (3.1),

$$
\phi_{-}(s)=\widetilde{\phi}_{-}(s)+G_{-}(\{-\infty\})
$$

and

$$
\phi_{+}(s)=\tilde{\phi}_{+}(s)+G_{+}(\{-\infty\}) .
$$

We have

$$
\begin{aligned}
& A(s) \\
= & A_{1}(s)+\widetilde{\phi}_{-}(s) G_{+}(\{\infty\})+\widetilde{\phi}_{+}(s) G_{-}(\{-\infty\})+G_{+}(\{\infty\}) G_{-}(\{-\infty\}),
\end{aligned}
$$

where

$$
A_{1}(s)=\int_{(-\infty, 0)} \widetilde{\phi}_{+}(-v) \frac{v}{s+v} G_{-}(d v)+\int_{(0, \infty)} \widetilde{\phi}_{-}(-u) \frac{u}{s+u} G_{+}(d u) .
$$

The function $A_{1}(s)$ is written as

$$
=\int_{(0, \infty)}^{A_{1}(s)} \int_{(-\infty, 0)} \frac{u v}{u-v}\left(\frac{1}{s+v}-\frac{1}{s+u}\right) G_{-}(d v) G_{+}(d u)
$$




$$
\begin{aligned}
& =\int_{(0, \infty)} \int_{(-\infty, 0)} \frac{u v}{(s+u)(s+v)} G_{-}(d v) G_{+}(d u) \\
& =\widetilde{\phi}_{+}(s) \widetilde{\phi}_{-}(s) .
\end{aligned}
$$

Hence we have $A(s)=\phi_{+}(s) \phi_{-}(s)=\mathscr{L} \mu(s)$. The proof is complete.

THEOREM 3.3. A sequence in $M E$ is shift compact if and only if it is conditionally compact.

Proof. Let $\left\{\mu_{n}\right\} \subset M E$ be a shift compact sequence. That is, there is a sequence $\left\{\gamma_{n}\right\} \subset \mathbf{R}$ such that $\left\{\mu_{n} * \delta_{r_{n}}\right\}$ is conditionally compact, where $\delta_{r_{n}}$ is the Dirac measure concentrated at $\gamma_{n}$. Let $\ell_{n}(y)$ be that density of the Lévy measure of $\mu_{n}$. Note that since

$$
\ell_{n}(y) \leqq \int_{0}^{\infty} e^{-|y| u} d u=|y|^{-1} \text { for } y \neq 0,
$$

the sequence $\left\{\int_{\mathbf{R}_{0}} \frac{y}{1+y^{2}} \ell_{n}(y) d y\right\}$ is bounded. We have

$$
\begin{aligned}
& \mathscr{L}\left(\mu_{n} * \delta_{\gamma_{n}}\right)(z)=\exp \left[i \gamma_{n} z+\int_{\mathbf{R}_{0}}\left(e^{i z y}-1\right) \ell_{n}(y) d y\right] \\
& \quad=\exp \left[i z\left\{\gamma_{n}+\int_{\mathbf{R}_{0}} \frac{y}{1+y^{2}} \ell_{n}(y) d y\right\}+\int_{\mathbf{R}_{0}}\left(e^{i z y}-1-\frac{i z y}{1+y^{2}} \ell_{n}(y) d y\right]\right.
\end{aligned}
$$

Hence $\left\{\gamma_{n}\right\}$ must be bounded. It follows that $\left\{\mu_{n}\right\}$ is conditionally compact. The converse is obvious.

\section{Class $C E$}

Let $C E_{+}^{f}$ be the class of $\mu \in \mathscr{P}\left(\mathbf{R}_{+}\right)$such that $\mathscr{L} \mu(s)=\prod_{k=1}^{m} a_{k}\left(s+a_{k}\right)^{-1}$ with $1 \leqq m<\infty$ and $0<a_{1}<a_{2}<\cdots<a_{m}$ and let $C E_{-}^{f}$ be the mirror image of $C E_{+}^{f}$. Let $C E^{f}=C E_{+}^{f} * C E_{-}^{f}$. We denote by $C E$ the closure of $C E^{f}$. Let $Z$ be the set of integers and set $Z_{0}=Z \backslash\{0\}$.

THEOREM 4.1. Let $\mu \in \mathscr{P}(\mathbf{R})$. Then, $\mu$ is a $C E$ distribution if and only if $\mu \in \mathscr{I}(\mathbf{R})$ and there is an $\mathbf{R}_{0}$-valued non-decreasing sequence $\left\{a_{k}\right\}_{k \in Z_{0} \cap I}$ for an interval I containing 0 such that

$$
\begin{aligned}
a_{k} & >0 \text { for } k>0, \\
& <0 \text { for } k<0,
\end{aligned}
$$




$$
\sum a_{k}^{-2}<\infty
$$

and the Lévy measure $\nu$ of $\mu$ is represented as

$$
\begin{array}{rlr}
\nu(d x) & =\left(x^{-1} \sum_{k>0} e^{-a_{k} x}\right) d x \quad \text { for } x>0, \\
& =\left(|x|^{-1} \sum_{k<0} e^{-a_{k} x}\right) d x & \text { for } x<0 .
\end{array}
$$

We call $\left\{a_{k}\right\}$ the parameter sequence of $\mu$.

Proof. Denote by $C E^{d}$ the subclass of $\mathscr{I}(\mathbf{R})$ consisting of distributions whose Lévy measure is of the form (4.3) satisfying conditions (4.1) and (4.2). The assertion of the theorem is that $C E=C E^{d}$. Let $\mu \in C E^{d}$ and let $\left\{a_{k}\right\}$ be its parameter sequence. Set

$$
q(x)=\sum_{k>0} 1_{\left(a_{k}, \infty\right)}(x)+\sum_{k<0} 1_{\left(-\infty, a_{k}\right)}(x),
$$

where $1_{A}(x)$ is the indicator function of a set $A$. Noting that $\left\{a_{k}\right\}$ is a monotone sequence, we have by (4.2) that $\int_{\mathbf{R}}|u|^{-3} q(u) d u<\infty$. It is easy to see that the Lévy measure $\nu$ of $\mu$ is written as

$$
\begin{aligned}
\nu(d x) & =\left(\int_{\mathbf{R}_{+}} e^{-x u} q(u) d u\right) d x \quad \text { for } x>0, \\
& =\left(\int_{\mathbf{R}_{-}} e^{-x u} q(u) d u\right) d x \text { for } x<0 .
\end{aligned}
$$

Hence, $\mu$ is a $B$ distribution with triplet $\left(\gamma, \sigma^{2}, q(x) d x\right)$ with some $\gamma$ and $\sigma^{2}$. Now we show that $\mu$ is approximated by $C E^{f}$-distributions. Let

$$
q_{1, n}(x)=\sum_{0<k \leq n} 1_{\left(a_{k}, \infty\right)}(x)+\sum_{0>k \geqq-n} 1_{\left(-\infty, a_{k}\right)}(x) q(x)
$$

and

$$
\gamma_{1, n}=\int_{\mathbf{R}_{+}} \frac{x}{1+x^{2}}\left\{\int_{\mathbf{R}_{+}} e^{-x u} q_{1, n}(u) d u\right\} d x+\int_{\mathbf{R}_{-}} \frac{x}{1+x^{2}}\left\{\int_{\mathbf{R}_{-}} e^{-x u} q_{1, n}(u) d u\right\} d x
$$

In case $\sigma^{2}>0$, set $\alpha_{n}=\left(2 n / \sigma^{2}\right)^{1 / 2}$ and let

$$
\begin{aligned}
& q_{2, n}(x)=0 \quad \text { for }|x|<\alpha_{n}, \\
& =n \quad \text { for }|x| \geqq \alpha_{n} \text {, }
\end{aligned}
$$

and choose $\beta_{n}>0$ so that

$$
\left(\gamma-\gamma_{1, n}\right) / \beta_{n} \rightarrow 0 \quad \text { as } n \rightarrow \infty
$$

and $\beta_{n}>\alpha_{n}$. In case $\sigma^{2}=0$, let 


$$
q_{2, n}(x) \equiv 0
$$

and choose $\beta_{n}>0$ so to satisfy (4.5). Let $\delta_{n}$ be the integral part of $\left\{\left|\gamma-\gamma_{1, n}\right| /\right.$ $\left.\int_{\mathbf{R}_{+}} \frac{1}{1+x^{2}} e^{-\beta n x} d x\right\}$ and let

$$
\begin{aligned}
\tilde{q}_{n}(x) & =0 & & \text { for } x<\beta_{n}, \\
& =\delta_{n} & & \text { for } x \geqq \beta_{n} .
\end{aligned}
$$

Define

$$
\begin{aligned}
q_{3, n}(x) & =\tilde{q}_{n}(x) \quad \text { if } \gamma>\gamma_{1, n} \\
& =\tilde{q}_{n}(-x) \text { if } \gamma \leqq \gamma_{1, n} .
\end{aligned}
$$

Let

$$
\gamma_{n}=\gamma_{1, n}+\operatorname{sign}\left(\gamma-\gamma_{1, n}\right) \delta_{n} \int_{\mathbf{R}_{+}} \frac{1}{1+x^{2}} e^{-\beta n x} d x
$$

Then, $Q_{n}(d x)=\left\{\sum_{j=1}^{3} q_{j, n}(x)\right\} d x$ satisfies (2.3). Let $\mu_{n}=\left(\gamma_{n}, 0, Q_{n}\right) \in B$. Since

$$
\gamma_{n}=\int_{\mathbf{R}_{+}} \frac{x}{1+x^{2}} d x \int_{(0, \infty)} e^{-x u} Q_{n}(d u)+\int_{\mathbf{R}_{-}} \frac{x}{1+x^{2}} d x \int_{(-\infty, 0)} e^{-x u} Q_{n}(d u),
$$

$\mu_{n}$ is approximated by $C E^{f}$-distributions. It is easy to see that $Q_{n}(I) \rightarrow$ $\int_{I} q(x) d x$ for every bounded interval $I$ in $\mathbf{R}$. We have by (4.2) that

$$
\begin{aligned}
& \lim _{M \rightarrow \infty} \limsup _{n \rightarrow \infty} 2 \int_{|u|>M} u^{-3} q_{1, n}(u) d u \\
= & \lim _{M \rightarrow \infty} \sum_{k} \frac{1}{\left(\left|a_{k}\right| \vee M\right)^{2}}=0 .
\end{aligned}
$$

We see by (4.6) that, for every $M$,

$$
\begin{aligned}
& \lim _{n \rightarrow \infty} 2 \int_{|u|>M}|u|^{-3}\left\{q_{2, n}(u)+q_{3, n}(u)\right\} d u \\
= & \lim _{n \rightarrow \infty}\left\{\sigma^{2}+\delta_{n} / \beta_{n}^{2}\right) \rightarrow \sigma^{2} .
\end{aligned}
$$

We have by (4.6) that

$$
\left|\gamma_{n}-\gamma\right| \leqq \int_{\mathbf{R}_{+}} \frac{1}{1+x^{2}} e^{-\beta_{n x}} d x \rightarrow 0 \text { as } n \rightarrow \infty .
$$

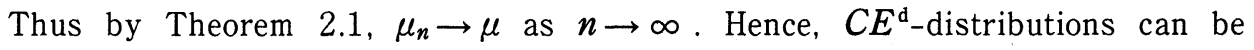
approximated by $C E^{f}$-distributions. Now we show that the class $C E^{d}$ is closed 
under weak convergence. Let $\mu_{n} \in C E^{d}$ and let $\mu_{n} \rightarrow \mu \in \mathscr{P}(\mathbf{R})$. Then, by Theorem 2.1, $\mu \in B$. Let $q_{n}$ be the density of $Q$-measure of $\mu_{n}$. Consider the convergence of the $Q$-measures on $(0, \infty)$. Since $q_{n}$ is a nondecreasing function, $Q$-measure of $\mu$ is absolutely continuous, its density $q$ is nondecreasing. Moreover, $q_{n}(x)$ converges to $q(x)$ at every continuity point of $q$. Noting that $q_{n}$ is a step function of step size 1 , we have that $q$ is also a step function with step size being positive integers. The same argument yields that the $Q$-measure of $\mu$ has a density $q$ also on $(-\infty, 0)$ and that $q$ is a nonincreasing step function on $(-\infty, 0)$ with step size being negative integers. By (2.3), $q(x)=0$ near $x=0$. Hence the class $C E^{d}$ is closed. Hence $C E^{d}=C E$.

Remark 4.1. The condition (4.2) for the parameter sequence $\left\{a_{n}\right\}$ of $\mu \in C E$ is equivalent to

$$
\int_{|x|<1} x^{2} \nu(d x)<\infty
$$

for the Lévy measure $\nu$ of $\mu$.

Remark 4.2. (i) A measure $\nu$ of the form (4.3) with subsidiary conditions (4.1) and (4.2) satisfies $\int_{|x|>1}|x| \nu(d x)<\infty$. Hence, for a $C E$ distribution, instead of (2.2) we can use another representation of its characteristic function. Let $\mu \in C E$ with canonical representation $\left[\gamma, \sigma^{2}, \nu\right]$. Then its characteristic function is represented as

$$
\begin{aligned}
& \mathscr{F} \mu(z) \\
& =\exp \left\{i \gamma^{\prime} z-\sigma^{2} z^{2} / 2+\int_{\mathbf{R}_{0}}\left(e^{i x z}-1-i z x\right) \nu(d x)\right\}
\end{aligned}
$$

Here

$$
\gamma^{\prime}=\gamma+\int_{\mathbf{R}_{0}} x^{3}\left(1+x^{2}\right)^{-1} \nu(d x)
$$

We call (4.7) the modified representation of $\mu \in C E$. We denote the modified representation of $\mu$ by $\left\{\gamma^{\prime}, \sigma^{2}, \nu\right\}$ or $\left\{\gamma^{\prime}, \sigma^{2},\left\{a_{j}\right\}\right\}$, where $\left\{a_{j}\right\}$ is the parameter sequence of $\mu$. Using this representation, as is shown in the next theorem, we can represent the Laplace transforms of $C E$ distributions as rather simple products.

(ii) Let $\mu_{n} \in C E$ and let $\left[\gamma_{n}, \sigma_{n}^{2}, \nu_{n}\right]$ and $\left\{\gamma_{n}^{\prime}, \sigma_{n}^{2}, \nu_{n}\right]$ be the canonical and the modified representations of $\mu_{n}$, respectively. If $\left[\gamma_{n}, \sigma_{n}{ }^{2}, \nu_{n}\right]$ satisfies the condition of Theorem A with $\mu=\left[\gamma, \sigma^{2}, \nu\right]=\left\{\gamma^{\prime}, \sigma^{2}, \nu\right\}$, then 


$$
\lim _{\varepsilon \downarrow 0} \limsup _{n \rightarrow \infty} \int_{|x|<\varepsilon}|x|^{3} \nu_{n}(d x)=0 .
$$

Hence by Theorem A, $\gamma_{n}^{\prime} \rightarrow \gamma^{\prime}$ as $n \rightarrow \infty$. The converse is also valid. Hence for $C E$ distributions, the condition (iii) of Theorem 2.1 can be replaced $\lim _{n \rightarrow \infty} \gamma_{n}{ }^{\prime}=\gamma^{\prime}$.

THEOREM 4.2. A distribution $\mu$ is a $C E$ distribution if and only if there are $\gamma^{\prime} \in \mathbf{R}, \sigma^{2} \geqq 0$ and an $\mathbf{R}_{0}$-valued non-decreasing sequence $\left\{a_{n}\right\}_{n \in Z_{0} \cap I}$ for an interval I containing 0 such that (4.1) and (4.2) are satisfied and the Laplace transform of $\mu$ is represented as

$$
\mathscr{L} \mu(s)=\exp \left(-\gamma^{\prime} s+\sigma^{2} s^{2} / 2\right) \prod_{n} a_{n}\left(s+a_{n}\right)^{-1} e^{a_{n}^{-1} s}
$$

for $-a_{1}<\operatorname{Re} s<-a_{-1}$.

Proof. Let $s=x+i y$. Note that

$$
\begin{aligned}
& \log \left(\left|\left(1+s / a_{n}\right) e^{-a_{n}^{-1} s}-1\right|+1\right) \\
\leqq & \left|\left(1+s / a_{n}\right) e^{-a_{n}^{-1} s}-1\right| \\
\leqq & \left|e^{-a_{n}^{-1} s}-1+s / a_{n}\right|+\left|s / a_{n}\right|\left|e^{-a_{n}^{-1} s}-1\right| \\
\leqq & \left|s / a_{n}\right|{ }^{2} R^{-2}(1+R) e^{R} \quad \text { for }\left|s / a_{n}\right|<R .
\end{aligned}
$$

Hence by (4.2), it is easy to see that the right hand side of (4.10) is convergent for $-a_{1}<\operatorname{Re} s<-a_{-1}$. For $s=-i z, z \in \mathbf{R}$, it is equal to

$$
\exp \left(i \gamma^{\prime} z-\sigma^{2} z^{2} / 2\right) \prod_{n} a_{n}\left(-i z+a_{n}\right)^{-1} e^{-i a_{n}^{-1} z}
$$

We can rewrite the above formula as

$$
\begin{aligned}
& \exp \left\{i \gamma^{\prime} z-\sigma^{2} z^{2} / 2+\sum\left[\log \left\{a_{n}\left(-i z+a_{n}\right)^{-1}\right\}-i a_{n}^{-1} z\right]\right\} \\
& =\exp \left\{i \gamma^{\prime} z-\sigma^{2} z^{2} / 2+\sum_{n>0} \int_{0}^{\infty}\left(e^{i z x}-1-i z x\right) x^{-1} e^{-a_{n} x} d x+\right. \\
& \left.+\sum_{n<0} \int_{-\infty}^{0}\left(e^{i z x}-1-i z x\right)|x|^{-1} e^{-a n x} d x\right\} \\
& =\exp \left\{i \gamma^{\prime} z-\sigma^{2} z^{2} / 2+\int_{0}^{\infty}\left(e^{i z x}-1-i z x\right)\left[\sum_{n>0} x^{-1} e^{-a_{n} x}\right] d x+\right. \\
& \left.+\int_{-\infty}^{0}\left(e^{i z x}-1-i z x\right)\left[\sum_{n<0}|x|^{-1} e^{-a_{n} x}\right] d x\right\} .
\end{aligned}
$$


Here we choose the branch of the logarithm so the argument is between $-\pi$ and $\pi$. On the other hand, $\int e^{s x} \mu(d x)$ is finite if $-a_{1}<\operatorname{Re} s<-a_{-1}$. This shows the validity of Theorem 4.2 .

The above representation shows that the class of densities of $C E$ distributions coincides with the class of $P F$ densities defined in Karlin [2] p. 335.

The quantities $\gamma^{\prime}$ appearing in (4.10) and (4.8) are identical. Write the closures of $C E_{+}^{f}$ and $C E_{-}^{f}$ as $C E_{+}$and $C E_{-}$, respectively. It is easy to show that the class $C E_{+}$coincides with the class $C E_{+}$defined in [8] and the class $C E_{+}$ (resp. $C E_{-}$) coincides with the class of $C E$ distributions with supports in $\mathbf{R}_{+}$ (resp. $\mathbf{R}_{-}$).

\section{Class $C M E$}

In [8], the class $C M E_{+}$is defined by $C M E_{+}=M E_{+} * C E_{+}$and it is proved that the class $C M E_{+}$is the closure of $C M E_{+}^{f}$. Let $C M E_{-}=M E_{-} * C E_{-}$. Then, the class $C M E_{-}$is the closure of $C M E_{-}^{f}$. We denote by $C M E$ the closure of $C M E^{f}$. This class contains both $C M E_{+}$and $C M E_{-}$. Define $M E_{+}^{f}$ as follows: $\mu \in$ $M E_{+}^{f}$ if and only if $\mu \in M E_{+}$and the mixing distribution $G$ of $\mu$ is supported on a finite number of points in $(0, \infty]$. Let $M E_{-}^{f}$ be the mirror image of $M E_{+}^{f}$ and let $M E^{f}=M E_{+}^{f} * M E_{-}^{f}$.

\section{THEOREM 5.1. $C M E=C E * M E$.}

Proof. By definition $C E$ is the closure of $C E^{f}$. It is easy to see that $M E$ is the closure of $M E^{f}$. Hence we have $C M E^{f} \subset C E * M E \subset C M E$. Now we show that $C E * M E$ is closed, which will prove the theorem. Let $\left\{\mu_{n}\right\}$ be a sequence in $C E * M E$ converging to a distribution $\mu$. Let $\mu_{n}^{1} \in C E$ and $\mu_{n}^{2} \in M E$ be such that $\mu_{n}=\mu_{n}^{1} * \mu_{n}^{1}$, for $n=1,2, \ldots$. Since the components $\left\{\mu_{n}^{1}\right\}$ and $\left\{\mu_{n}^{2}\right\}$ are both shift compact, $\left\{\mu_{n}^{2}\right\}$ is conditionally compact by Theorem 3.3 . Hence $\left\{\mu_{n}^{1}\right\}$ is also conditionally compact. Now we can choose a subsequence $n^{\prime}$ so that $\mu_{n^{\prime}}^{1} \rightarrow \mu^{1}$ $\in C E$ and $\mu_{n^{\prime}}^{2} \rightarrow \mu^{2} \in M E$ as $n^{\prime} \rightarrow \infty$ and we have

$$
\mu=\mu^{1} * \mu^{2}
$$

Hence, $C E * M E$ is closed.

Remark 5.1. A distribution $\mu \in C M E$ is determined by the modified representation $\left\{\boldsymbol{\gamma}, \sigma^{2}, \boldsymbol{a}=\left\{a_{j}\right\}\right\}$ of its $C E$ component and the $Q$-measure $Q$ of its $M E$ 
component. Let us call $\left(\gamma, \sigma^{2}, \boldsymbol{a}, Q\right)$ the quadruplet of the $C M E$ distribution $\mu$. Since there are many ways of decomposing $\mu$ as $\mu=\mu_{1} * \mu_{2}$ with $\mu_{1} \in C E$ and $\mu_{2} \in M E$, there are many quadruplets that determine $\mu$. But, among them, there is a unique decomposition which maximizes the density of the $Q$-measure of $\mu_{1}$. Choosing $\mu_{1} \in C E$ and $\mu_{2} \in M E$ in this way, the quadruplet $\left(\gamma^{\prime}, \sigma^{2}, \boldsymbol{a}, Q\right)$ is uniquely determined by $\mu$. In the following, by qudruplet of $\mu$, we always mean this quadruplet.

The parameter sequence $\boldsymbol{a}=\left\{a_{j}\right\}_{j \in Z_{0} \cap I}$ may possibly be empty. In case $a_{j}$ is not defined, we regard $a_{j}=\infty$ if $j>0$ and $a_{j}=-\infty$ if $j<0$.

\section{Representation of Laplace transforms of distributions of classes $M E_{+}^{d}$ and $C M E_{+}^{d}$}

We say that a distribution on $(0, \infty]$ is discrete if its support is a finite or countably infinite set which has no accumulation point in $[0, \infty)$. A distribution $\mu$ on $\mathbf{R}_{+}$is said to belong to class $M E_{+}^{d}$ if $\mu$ belongs to $M E_{+}$and its mixing distribution is discrete.

THEOREM 6.1. Let $\left\{a_{j}\right\}$ and $\left\{\beta_{j}\right\}$ be sequences of positive real numbers such that $0<\alpha_{1}<\beta_{1}<\alpha_{2}<\beta_{2}<\cdots$ and $\alpha_{j}, \beta_{j} \rightarrow \infty$ as $j \rightarrow \infty$. Then the infinite product

$$
f(s)=\prod_{j=1}^{\infty}\left(1+\frac{s}{\beta_{j}}\right) /\left(1+\frac{s}{\alpha_{j}}\right)
$$

absolutely and uniformly converges on each compact set in $\mathbf{C} \backslash\left\{-a_{1},-\alpha_{2}, \ldots\right\}$ and there is $\mu \in M E_{+}^{d}$ such that

$$
\mathscr{L}_{\mu}(s)=f(s) \text { for } s>0 .
$$

Moreover, $\mathscr{L} \mu(s)$ is written as

$$
\mathscr{L} \mu(s)=\exp \int_{0}^{\infty}\left(e^{-s x}-1\right)\left\{\int_{0}^{\infty} e^{-x u} q(u) d u\right\} d x,
$$

where

$$
\begin{array}{rlrl}
q(u) & =0 & 0 & <u<\alpha_{1}, \\
& =1 & & \alpha_{j}<u<\beta_{j}, \quad j=1,2, \ldots \\
& =0 & \beta_{j}<u<\alpha_{j+1}, j=1,2, \ldots
\end{array}
$$

Proof. First step. We show the absolute and uniform convergence of $f$ on each compact set in $\mathbf{C} \backslash\left\{-\alpha_{1},-\alpha_{2}, \cdots\right\}$. Set 
and

$$
a_{j}(s)=\left(1+\frac{s}{\beta_{j}}\right) /\left(1+\frac{s}{\alpha_{j}}\right)
$$

$$
b_{j}(s)=1-a_{j}(s) \text {. }
$$

Then we have

$$
b_{j}(s)=s\left(1+s / \alpha_{j}\right)^{-1}\left\{\left(\alpha_{j}\right)^{-1}-\left(\beta_{j}\right)^{-1}\right\}
$$

and the inside of the braces in (6.3) is positive. Let $D_{T}=\{s ;|s|<T\}$. If there is $i$ such that $\alpha_{i} \leqq T<\alpha_{i+1}$, then choose $M$ so that $1 / M<1 / T-1 / \alpha_{i+1}$. Then we get that, for $s \in D_{T}$ and for all $j \geqq i+1$,

$$
\begin{gathered}
\left|1 / s+1 / \alpha_{j}\right| \geqq|1 / s|-\left|1 / \alpha_{j}\right| \\
>1 / T-1 / \alpha_{j} \geqq 1 / T-1 / \alpha_{i+1}>1 / M .
\end{gathered}
$$

That is,

$$
\left|s /\left(1+s / \alpha_{j}\right)\right|<M .
$$

Moreover, $\left|b_{j}(s)\right|<1$ for large $j$, since $\alpha_{j}, \beta_{j} \rightarrow \infty$ as $j \rightarrow \infty$. We denote by $U_{T, \delta}$ the set $D_{T}$ with the $\delta$-neighborhoods of $-\alpha_{1}, \ldots,-\alpha_{i}$ excluded. Since $s /(1+$ $\left.s / \alpha_{j}\right)$ is bounded in $j$ and $s \in U_{T, \delta}$, there is $M>0$ such that

$$
\sum_{j=1}^{\infty}\left|b_{j}(s)\right| \leqq \sum_{j=1}^{\infty} M\left(1 / \alpha_{j}-1 / \alpha_{+1}\right) \leqq M / \alpha_{1}<\infty
$$

for $s \in U_{T, \delta}$. By this we have that $\sum_{j=1}^{\infty} b_{j}(s)$ converges absolutely and uniformly on any compact set in $\mathbf{C} \backslash\left\{-\alpha_{1},-\alpha_{2}, \ldots\right\}$. Hence the infinite product $f(s)$ converges absolutely and uniformly on any compact set in $\mathbf{C} \backslash\left\{-\alpha_{1},-\alpha_{2}, \ldots\right\}$.

Second step. We show that $f$ is the Laplace transform of the $M E_{+}^{d}$ distribution $\mu$ defined by (6.2). Note that

$$
f_{n}(s)=\prod_{j=1}^{n}\left(1+\frac{s}{\beta_{j}}\right) /\left(1+\frac{s}{\alpha_{j}}\right)
$$

is the Laplace transform of an $M E_{+}^{f}$ distribution $\mu_{n}$ (Steutel [5]). Moreover, $f_{n}$ is written as

$$
f_{n}(s)=\exp \int_{0}^{\infty}\left(e^{-s x}-1\right)\left\{\int_{0}^{\infty} e^{-x u} q_{n}(u) d u\right\} d x
$$

where

$$
\begin{aligned}
q_{n}(u) & =0 & & u<\alpha_{1} \\
& =1 & & a_{j}<u<\beta_{j} \quad j=1,2, \ldots, n, \\
& =0 & & \beta_{j}<u<\alpha_{j+1} \quad j=1,2, \ldots, n,
\end{aligned}
$$


Here we understand $\alpha_{n+1}=\infty$. We have

$$
q_{n}(u) d u \rightarrow q(u) d u \text { as } n \rightarrow \infty
$$

and

$$
\int_{M}^{\infty} \frac{q_{n}(u)}{u^{2}} d u \leqq 1 / M \rightarrow 0 \text { as } M \rightarrow \infty
$$

By the continuity theorem for $B_{+}$(Bondesson [1]), letting $\mu$ be the distribution with Laplace transform of the form (6.2), we have $\mu_{n} \rightarrow \mu$ as $n \rightarrow \infty$. Hence $f_{n}(s)$ $\rightarrow \mathscr{L} \mu(s)$ for $s>0$ as $n \rightarrow \infty$. On the other hand, $f_{n}(s)$ converges to $f(s)$ as $n \rightarrow \infty$ absolutely and uniformly on any compact set in $\mathbf{C} \backslash\left\{-\alpha_{1},-\alpha_{2}, \ldots\right\}$, $\mathscr{L} \mu(s)=f(s)$ should hold for $s>0$. By Theorem 3.1 , the mixing distribution $G_{n}$ of $\mu_{n}$ converges weakly to the mixing distribution $G$ of $\mu$ as a distribution on $(0, \infty]$. Since the support of $G_{n}$ is contained in $\left\{\alpha_{j}\right\}_{j=1}^{n} \cup\{\infty\}$, the support of $G$ is contained in $\left\{\alpha_{j}\right\}_{j=1}^{\infty} \cup\{\infty\}$. Hence $\mu \in M E_{+}^{d}$. The proof is complete.

THEOREM 6.2. Let $\left\{\alpha_{j}\right\}$ and $\left\{\beta_{j}\right\}$ be non-decreasing infinite sequences of positive real numbers satisfying $\alpha_{\imath} \neq \beta_{j}$ for all $i, j$. Let $\mu \in M E_{+}$such that

$$
\mathscr{L} \mu(s)=\prod_{j=1}^{\infty}\left(1+\frac{s}{\beta_{j}}\right) /\left(1+\frac{s}{\alpha_{j}}\right)
$$

for $s \geqq 0$. Then

$$
0<\alpha_{1}<\beta_{1}<\alpha_{2}<\beta_{2}<\cdots
$$

Moreover, if $\alpha_{j}, \beta_{j} \rightarrow \infty$ as $j \rightarrow \infty$, then $\mu \in M E_{+}^{d}$.

Proof. By the assumption,

$$
\begin{aligned}
\mathscr{L} \mu(s) & =\exp \left[\sum_{j=1}^{\infty}\left\{\log \frac{\alpha_{j}}{s+\alpha_{j}}-\log \frac{\beta_{j}}{s+\beta_{j}}\right\}\right] \\
& =\exp \left[\sum_{j=1}^{\infty} \int_{\alpha,}^{\beta_{j}} \frac{s}{u(s+u)} d u\right], \text { for } s \geqq 0 .
\end{aligned}
$$

Thus the density $q(u)$ of the $Q$-measure of $\mu$ is written as $q(u)=\sum_{j=1}^{\infty} 1_{\left[\alpha_{j}, \beta_{j}\right]}(u)$. We show (6.6) by induction. Remind that $q(u)$ is nonnegative and bounded by 1 a.e. Hence $\alpha_{1}<\beta_{1}$. Assume that

$$
0<\alpha_{1}<\beta_{1}<\alpha_{2}<\beta_{2}<\cdots<\alpha_{n}<\beta_{n}
$$

holds for $n \geqq 1$. If $\alpha_{n} \leqq \alpha_{n+1}<\beta_{n+1}$, then since $\beta_{n} \leqq \beta_{n+1}, q(u)=2$ on $\left(\alpha_{n+1}\right.$, $\left.\beta_{n}\right)$. This can not occur. Hence $\alpha_{n}<\beta_{n}<\alpha_{n+1}$. Since $q$ is nonnegative, $\beta_{n}<\beta_{n+1}$ $<\alpha_{n+1}$ can not occur. Hence $\alpha_{n}<\beta_{n}<\alpha_{n+1}<\beta_{n+1}$. The proof is complete. 
Theorem 6.3. Let $\mu \in M E_{+}^{d}$ and let $G$ be its mixing distribution. Suppose that $\left\{\alpha_{j}\right\}_{1 \leq j<\infty}=(\operatorname{supp} G) \backslash\{\infty\}$, where $\left\{\alpha_{j}\right\}$ is an infinite sequence increasing to $\infty$. Then there is a sequence of real numbers $\left\{\beta_{k}\right\}_{k=1}^{\infty}$ such that

$$
0<\alpha_{1}<\beta_{1}<\alpha_{2}<\beta_{2}<\cdots
$$

and

$$
\mathscr{L} \mu(s)=\prod_{j=1}^{\infty}\left(1+\frac{s}{\beta_{j}}\right) /\left(1+\frac{s}{\alpha_{j}}\right), s>0 .
$$

Proof. Let $p_{j}=G\left(\left\{\alpha_{j}\right\}\right)$ and $p_{\infty}=1-\sum_{j=1}^{\infty} p_{j}$. We have

$$
\mathscr{L} \mu(s)=p_{\infty}+\sum_{j=1}^{\infty} \frac{\alpha_{j}}{s+\alpha_{j}} p_{j} \text { for } s>0 .
$$

Denote by $f(s)$ the right hand side of (6.7). Set $P=\left\{-\alpha_{j}\right\}_{j=1}^{\infty}$. Then the analytic continuation of $f$ to $\mathbf{C} \backslash P$ is unique and $f$ is a meromorphic function. Every pole of $f$ has degree 1 and the set of poles coincides with $P$. The function $f$ is term-wise differentiable in $\mathbf{C} \backslash P$ and

$$
f^{\prime}(s)=-\sum_{j=1}^{\infty} \frac{\alpha_{j}}{\left(s+\alpha_{j}\right)^{2}} p_{j}
$$

This shows that $f$ is decreasing in every interval in $\mathbf{R} \backslash P$ and the set of zeros $Z=\left\{-\beta_{j}\right\}_{j \geqq 1}$ of $f$ in $\mathbf{R} \backslash P$ satisfies

$$
\cdots<-\beta_{2}<-\alpha_{2}<-\beta_{1}<-\alpha_{1}<0 .
$$

Set $s=a+b i$. Since

$$
f(s)=p_{\infty}+\sum_{j=1}^{\infty} \frac{\alpha_{j}\left(a+\alpha_{j}\right)}{\left(a+\alpha_{j}\right)^{2}+b^{2}} p_{j}+i \sum_{j=1}^{\infty} \frac{-\alpha_{j} b}{\left(a+\alpha_{j}\right)^{2}+b^{2}} p_{j},
$$

the imaginary part of $f(s)$ vanishes if and only if $b=0$. Hence $f$ does not have zero points outside $\mathbf{R}$. Set

$$
\begin{aligned}
E(u, n) & =1-u & & \text { for } n=0, \\
& =(1-u) \exp \left\{\sum_{k=1}^{n} \frac{u^{k}}{k}\right\} & & \text { for } n=1,2, \ldots
\end{aligned}
$$

Define a function $\varphi$ by

$$
\varphi(s)=\prod_{j=1}^{\infty} E\left(-\frac{s}{\alpha_{j}}, j\right)
$$

Then, since $\sum_{j=1}^{\infty}\left(\frac{T}{\alpha_{j}}\right)^{j}<\infty$ for arbitrary $T>0, \varphi$ is an entire function and the set of zero points of $\varphi$ coincides with $P$ ([3] p. 233). Let

$$
\varphi_{0}(s)=\varphi(s) f(s) .
$$


Then $\varphi_{0}$ is an entire function with the set of zero points coinciding with $Z$. By Weierstrass's Factorization Theorem ([3] p. 234), there is an entire function $g_{0}$ such that $\varphi_{0}$ can be written as

Hence,

$$
\varphi_{0}(s)=e^{g_{0}(s)} \prod_{j=1}^{\infty} E\left(-\frac{s}{\beta_{j}}, j\right) .
$$

$$
f(s)=e^{g_{0}(s)} \prod_{j=1}^{\infty} E\left(-\frac{s}{\beta_{j}}, j\right) / \prod_{j=1}^{\infty} E\left(-\frac{s}{\alpha_{j}}, j\right) .
$$

This yields

$$
f(s)=e^{g_{0}(s)} \prod_{j=1}^{\infty}\left(1+\frac{s}{\beta_{j}}\right) /\left(1+\frac{s}{\alpha_{j}}\right) \exp \left[\sum_{k=1}^{j} \frac{(-s)^{k}}{k}\left\{\left(\beta_{j}\right)^{-k}-\left(\alpha_{j}\right)^{-k}\right\}\right] .
$$

We have, for any positive integer $M$,

$$
\begin{aligned}
& \prod_{j=1}^{M}\left(1+\frac{s}{\beta_{j}}\right) /\left(1+\frac{s}{\alpha_{j}}\right) \exp \left[\sum_{k=1}^{j} \frac{(-s)^{k}}{k}\left\{\left(\beta_{j}\right)^{-k}-\left(\alpha_{j}\right)^{-k}\right\}\right] \\
= & \left\{\prod_{j=1}^{M}\left(1+\frac{s}{\beta_{j}}\right) /\left(1+\frac{s}{\alpha_{j}}\right)\right\} \exp \left[\sum_{j=1}^{M} \sum_{k=1}^{j} \frac{(-s)^{k}}{k}\left\{\left(\beta_{j}\right)^{-k}-\left(\alpha_{j}\right)^{-k}\right\}\right] .
\end{aligned}
$$

If $|s|<\alpha_{N}$ and $M>N$, then

$$
\begin{aligned}
& \sum_{j=N+1}^{M} \sum_{k=1}^{j} \frac{|s|^{k}}{k}\left\{\left(\alpha_{j}\right)^{-k}-\left(\beta_{j}\right)^{-k}\right\} \\
\leqq & \sum_{k=1}^{\infty} \sum_{j=N+1}^{M} \frac{|s|^{k}}{k}\left\{\left(\alpha_{j}\right)^{-k}-\left(\alpha_{j+1}\right)^{-k}\right\} \\
\leqq & \sum_{k=1}^{\infty} \frac{1}{k}\left(|s| / \alpha_{N}\right)^{k}<\infty .
\end{aligned}
$$

It follows that

$$
g_{1}(s)=\sum_{j=1}^{\infty} \sum_{k=1}^{j} \frac{(-s)^{k}}{k}\left\{\left(\beta_{j}\right)^{-k}-\left(\alpha_{j}\right)^{-k}\right\}
$$

is an entire function. By Theorem 6.2,

$$
\prod_{j=1}^{\infty}\left(1+\frac{s}{\beta_{j}}\right) /\left(1+\frac{s}{\alpha_{j}}\right)
$$

is a meromorphic function. Hence $f(s)$ is written as

$$
f(s)=e^{g(s)} \prod_{j=1}^{\infty}\left(1+\frac{s}{\beta_{j}}\right) /\left(1+\frac{s}{\alpha_{j}}\right),
$$

where $g(s)=g_{0}(s)+g_{1}(s)$ is an entire function. For $s>0$ let $A(s)=\log f(s)$ and $B(s)=\log \prod_{j=1}^{\infty}\left(1+\frac{s}{\beta_{j}}\right) /\left(1+\frac{s}{\alpha_{j}}\right)$. Since $f(s)=\mathscr{L} \mu(s)$ for $s>0$, we have

$$
A(s)=\int_{0}^{\infty}\left(e^{-s x}-1\right)\left\{\int_{0}^{\infty} e^{-x u} q(u) d u\right\} d x
$$


for $s>0$ where $0 \leqq q(u) \leqq 1$ a.e. and $\int_{0}^{1} u^{-1} q(u) d u<\infty$. Let

$$
\begin{aligned}
& q_{1}(u)=0 \text { for } 0<\alpha_{1} \text {, } \\
& =1 \text { for } \alpha_{j}<u<\beta_{j} \quad j=1,2, \ldots, \\
& =0 \text { for } \beta_{j}<u<\alpha_{j+1} \quad j=1,2, \ldots .
\end{aligned}
$$

Since, by Theorem 6.1 ,

$$
B(s)=\int_{0}^{\infty}\left(e^{-s x}-1\right)\left\{\int_{0}^{\infty} e^{-x u} q_{1}(u) d u\right\} d x,
$$

for $s>0$ we have

$$
g(s)=A(s)-B(s)+C
$$

and

$$
A(s)-B(s)=\int_{0}^{\infty} \frac{s}{(s+u) u}\left(q_{1}(u)-q(u)\right) d u,
$$

where $C$ is a constant satisfying $e^{c}=1$. Since $(A(s)-B(s)) / s$ is the Stieltjes transform of $\left(q_{1}(x)-q(x)\right) x^{-1} d x,\left(q_{1}(x)-q(x)\right) x^{-1}$ is obtained by the inversion formula for Stieltjes transform. Since $g(s)$ is an entire function, $\left(q_{1}(x)-\right.$ $q(x)) x^{-1} d x$ can not have a mass in $(0, \infty)$. Hence

$$
q_{1}(x)-q(x)=0 \text { a.e. }
$$

and $g(s)$ is a constant $C$. Hence, we have

$$
\mathscr{L} \mu(s)=\prod_{j=1}^{\infty}\left(1+\frac{s}{\beta_{j}}\right) /\left(1+\frac{s}{\alpha_{j}}\right) .
$$

The proof is complete.

Remark 6.1. Let $\mu \in M E_{+}^{\text {d }}$ and let $G$ be its mixing distribution. Let $\mathscr{L} \mu(s)$

$$
\begin{aligned}
& =\prod_{j=1}^{\infty}\left(1+\frac{s}{\beta_{j}}\right) /\left(1+\frac{s}{\alpha_{j}}\right) \text {. Then } \\
& \qquad G(\{\infty\})=\prod_{j=1}^{\infty} \alpha_{j} / \beta_{j}
\end{aligned}
$$

Proof. Let $Q$ be the $Q$-measure of $\mu$. Since, by Remark 3.1,

$$
G(\{\infty\})=\exp \left\{-\int_{0}^{\infty} \frac{1}{u} Q(d u)\right\}
$$

and since $-\int_{0}^{\infty} \frac{1}{u} Q(d u)=\sum_{j=1}^{\infty} \log \left(\alpha_{j} / \beta_{j}\right)$, we get the conclusion. 
Denote $C M E_{+}^{d}=C E_{+} * M E_{+}^{d}$.

THEOREM 6.4. Let $\mu \in C M E_{+}$. Suppose that its Laplace transform is represented as

$$
\mathscr{L} \mu(s)=\prod_{j=1}^{\infty}\left(1+\frac{s}{\beta_{j}}\right) /\left(1+\frac{s}{\alpha_{j}}\right)
$$

where $\left\{\alpha_{j}\right\},\left(\beta_{j}\right\}$ are disjoint divergent non-decreasing sequences of positive reals satisfying $\alpha_{j} \neq \beta_{j}$ for all $i, j$. Then,

(i) there is a subsequence $\left\{\alpha_{n j}\right\}$ of $\left\{\alpha_{j}\right\}$ such that

$$
0<\alpha_{n_{1}}<\beta_{1}<\alpha_{n_{2}}<\beta_{2}<\cdots
$$

and

(ii) $\sum_{\gamma \in \Gamma} \gamma^{-1}<\infty$ for $\Gamma=\left\{\alpha_{j}\right\}_{j=1}^{\infty} \backslash\left\{\alpha_{n j}\right\}_{j=1}^{\infty}$.

Hence $\mu \in C M E_{+}^{d}$.

Proof. If $\mu \in C M E_{+}$, then there is $\mu_{1} \in C E_{+}, \mu_{2} \in M E_{+}$such that $\mu=$ $\mu_{1} * \mu_{2}$ and there is a finite or infinite sequence $0<\gamma_{1} \leqq \gamma_{2} \leqq \cdots$ s

$$
\begin{gathered}
\mathscr{L} \mu_{1}(s)=\prod_{j=1}^{\infty} 1 /\left(1+\frac{s}{\gamma_{j}}\right), \\
\sum 1 / \gamma_{n}<\infty .
\end{gathered}
$$

See [8]. Hence,

$$
\mathscr{L} \mu_{2}(s)=\prod_{j=1}^{\infty}\left(1+\frac{s}{\delta_{j}}\right) /\left(1+\frac{s}{\tau_{j}}\right)
$$

where

$$
\begin{aligned}
\left\{\delta_{j}\right\}=\left\{\beta_{j}\right\} & \cup\left(\left\{\gamma_{j}\right\} \backslash\left\{\alpha_{j}\right\}\right),\left\{\tau_{j}\right\}=\left\{\alpha_{j}\right\} \backslash\left\{\gamma_{j}\right\}, \\
& 0<\tau_{1} \leqq \tau_{2} \leqq \cdots, \\
& 0<\delta_{1} \leqq \delta_{2} \leqq \cdots .
\end{aligned}
$$

We may assume that $\left\{\tau_{j}\right\}$ is an infinite sequence. Then $\delta_{j}, \tau_{j} \rightarrow \infty$ as $j \rightarrow \infty$. By Theorems 6.1 and 2, we have

$$
0<\tau_{1}<\delta_{1}<\tau_{2}<\delta_{2}<\cdots
$$

and $\mu_{2} \in M E_{+}^{d}$. Hence $\mathscr{L} \mu(s)$ can be analytically continued to $\mathbf{C} \backslash\left\{-\alpha_{1}\right.$, $\left.-\alpha_{2}, \ldots\right\}$ and zero points of analytic continuation of $\mathscr{L} \mu(s)$ are contained in $\left\{\beta_{j}\right\}$. We have $\left\{\gamma_{j}\right\} \subset\left\{\alpha_{j}\right\},\left\{\delta_{j}\right\}=\left\{\beta_{j}\right\}$ and we have (i) and (ii). 


\section{REFERENCES}

[1] Bondesson, L., Classes of infinitely divisible distributions and densities, $Z$. Wahrsch. Verw. Gebiete, 57 (1981), 39-71.

[2] Karlin, S., Total Positivity, Vol. 1, Stanford Univ. Stanford, 1968.

[3] Nevanlinna, R., Paatero, V., Introduction to complex analysis, Addison-Wesley, Reading-Menlo Park-London-Don Mills, 1969.

[4] Sato, K., On zeros of a system of polynomials and application to sojourn time distributions of birth-and-death processes, Trans. Amer. math. Soc., 309 (1988), $375-390$.

[5] Steutel, F. W., Preservation of Infinite Divisibility under Mixing and Related Topics, Math. Centre Tracts, 33, Amsterdam, 1970.

[6] Thorin O., An extension of the notion of a generalized $\Gamma$-convolution, Scand. Actuarial J., (1978), 141-149.

[7] Yamazato, M., Strongly unimodal infinitely divisible distributions, Ann. Probab., 10 (1982), 589-601.

[8] - Characterization of the class of upward passage time distributions of birth and death processes and related results, J. Math. Soc. Japan, 40 (1988), 477-499.

[9] - Hitting time distributions of single points for 1-dimensional generalized diffusion processes, Nagoya Math. J., 119 (1990).

[10] - Strong unimodality of infinitely divisible distributions of class $C M E$, submitted.

Departmert of Mathemtics

Nagoya Institute of Technology

Showa-ku, Nagoya 466

Japan 